\title{
New Exact Explicit Nonlinear Wave Solutions for the Broer-Kaup Equation
}

\author{
Zhenshu Wen \\ School of Mathematical Sciences, Huaqiao University, Quanzhou 362021, China \\ Correspondence should be addressed to Zhenshu Wen; wenzhenshu@hqu.edu.cn \\ Received 27 November 2013; Accepted 10 January 2014; Published 20 February 2014 \\ Academic Editor: Yongkun Li
}

Copyright (c) 2014 Zhenshu Wen. This is an open access article distributed under the Creative Commons Attribution License, which permits unrestricted use, distribution, and reproduction in any medium, provided the original work is properly cited.

We study the nonlinear wave solutions for the Broer-Kaup equation. Many exact explicit expressions of the nonlinear wave solutions for the equation are obtained by exploiting the bifurcation method and qualitative theory of dynamical systems. These solutions contain solitary wave solutions, singular solutions, periodic singular solutions, and kink-shaped solutions, most of which are new. Some previous results are extended.

\section{Introduction}

In 1975, Broer [1] obtained a dispersive equation as follows:

$$
\begin{gathered}
u_{t}+\eta_{x}+\frac{1}{2}\left(u^{2}\right)_{x}=0, \\
\eta_{t}+\left(u \eta+u+u_{x x}\right)_{x}=0,
\end{gathered}
$$

which describes the evolution of horizontal velocity component $u(x, t)$ of water waves of height $\eta(x, t)$ propagating in both directions in an infinite narrow channel of finite constant depth. Equation (1) plays an important role in nonlinear physics and gains considerate attention [2-4]. The traveling wave solutions for (1) have been studied by many works, such as [5-10].

In this paper, we employ the bifurcation method and qualitative theory of dynamical systems [11-21] to investigate the nonlinear wave solutions for (1), and we obtain many exact explicit expressions of nonlinear wave solutions for (1). These nonlinear wave solutions contain solitary wave solutions, singular solutions, periodic singular solutions, and kink-shaped solutions, most of which, to our knowledge, are newly obtained.

The remainder of this paper is organized as follows. In Section 2, we show the bifurcation of phase portraits corresponding to (1). We state our main results and the theoretical derivation for the main results in Section 3. A short conclusion will be given in Section 4 .

\section{Bifurcation of Phase Portraits}

In this section, we give the process of obtaining the bifurcation of phase portraits corresponding to (1).

For given constant $c$, substituting $u(x, t)=\varphi(\xi), \eta(x, t)=$ $\psi(\xi)$ with $\xi=x-c t$ into (1), it follows

$$
\begin{gathered}
-c \varphi^{\prime}+\psi^{\prime}+\frac{1}{2}\left(\varphi^{2}\right)^{\prime}=0, \\
-c \psi^{\prime}+\left(\psi \varphi+\varphi+\varphi^{\prime \prime}\right)^{\prime}=0 .
\end{gathered}
$$

Integrating (2) once leads to

$$
\begin{gathered}
-c \varphi+\psi+\frac{1}{2} \varphi^{2}=g, \\
-c \psi+\psi \varphi+\varphi+\varphi^{\prime \prime}=G,
\end{gathered}
$$

where both $g$ and $G$ are integral constants, respectively.

From the first equation of system (3), we obtain

$$
\psi=g+c \varphi-\frac{1}{2} \varphi^{2} .
$$

Substituting (4) into the second equation of system (3) leads to

$$
\varphi^{\prime \prime}=\frac{1}{2} \varphi^{3}-\frac{3 c}{2} \varphi^{2}+\left(c^{2}-g-1\right) \varphi+c g+G .
$$



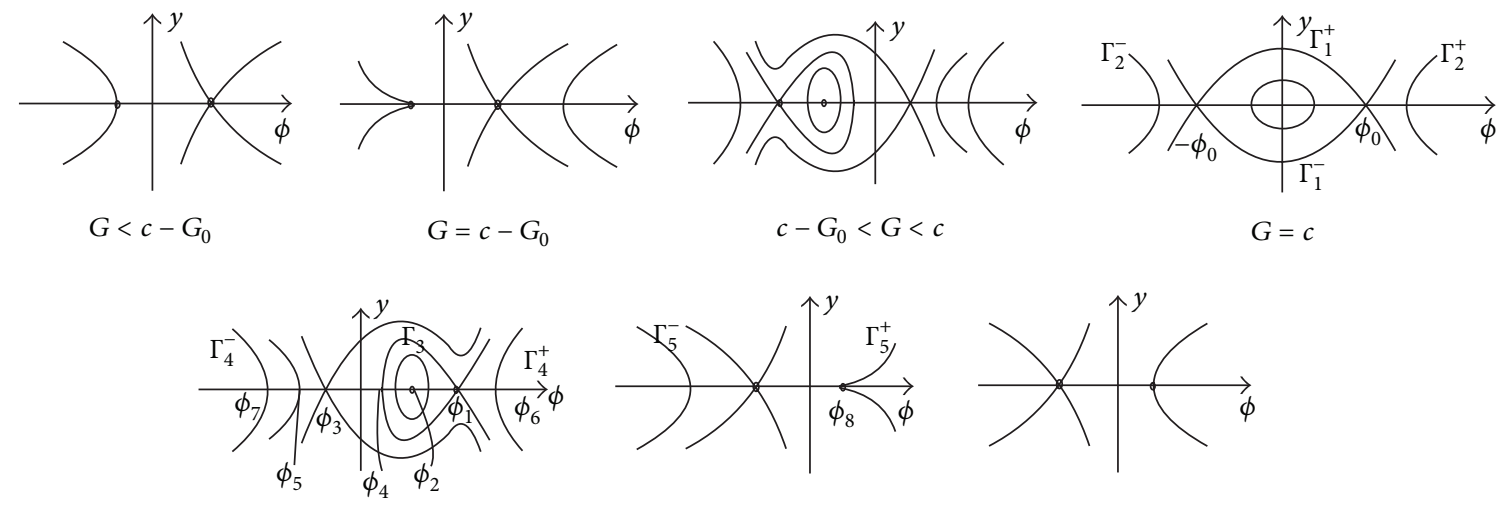

$c<G<G_{0}+c$

$G=G_{0}+c$

$G>G_{0}+c$

FIGURE 1: The phase portraits of system (7).

By setting $\varphi=\phi+c$, (5) becomes

$$
\phi^{\prime \prime}=\frac{1}{2} \phi^{3}-\frac{1}{2}\left(c^{2}+2 g+2\right) \phi+G-c
$$

Letting $y=\phi^{\prime}$, we obtain a planar system

$$
\begin{gathered}
\frac{\mathrm{d} \phi}{\mathrm{d} \xi}=y, \\
\frac{\mathrm{d} y}{\mathrm{~d} \xi}=\frac{1}{2} \phi^{3}-\frac{1}{2}\left(c^{2}+2 g+2\right) \phi+G-c,
\end{gathered}
$$

with first integral

$$
H(\phi, y)=\frac{1}{2} y^{2}-\frac{1}{8} \phi^{4}+\frac{1}{4}\left(c^{2}+2 g+2\right) \phi^{2}-(G-c) \phi .
$$

Now, we study the bifurcation of phase portraits of system (7). Set

$$
\begin{gathered}
f_{0}(\phi)=\frac{1}{2} \phi^{3}-\frac{1}{2}\left(c^{2}+2 g+2\right) \phi \\
f(\phi)=\frac{1}{2} \phi^{3}-\frac{1}{2}\left(c^{2}+2 g+2\right) \phi+G-c .
\end{gathered}
$$

Obviously, $f_{0}(\phi)$ has three zero points, which can be expressed as

$$
\phi=0, \pm \phi_{0}
$$

where $\phi_{0}=\sqrt{c^{2}+2 g+2}$, when $c^{2}+2 g+2>0$.

Additionally, it is easy to obtain the two extreme points of $f(\phi)$ as follows:

$$
\phi_{ \pm}^{*}= \pm \sqrt{\frac{1}{3}\left(c^{2}+2 g+2\right)} .
$$

Let

$$
g_{0}=\left|f_{0}\left(\phi_{ \pm}^{*}\right)\right|=\sqrt{\frac{\left(c^{2}+2 g+2\right)^{3}}{27}}
$$

which is the absolute value of extreme values of $f_{0}(\phi)$.

Let $\left(\phi_{i}, 0\right)$ be one of the singular points of system (7). Then the characteristic values of the linearized system of system (7) at the singular point $\left(\phi_{i}, 0\right)$ are

$$
\lambda_{ \pm}= \pm \sqrt{f^{\prime}\left(\phi_{i}\right)}
$$

From the qualitative theory of dynamical systems, we therefore know that

(i) if $f^{\prime}\left(\phi_{i}\right)>0$, then $\left(\phi_{i}, 0\right)$ is a saddle point;

(ii) if $f^{\prime}\left(\phi_{i}\right)<0$, then $\left(\phi_{i}, 0\right)$ is a center point;

(iii) if $f^{\prime}\left(\phi_{i}\right)=0$, then $\left(\phi_{i}, 0\right)$ is a degenerate saddle point.

Therefore, based on the above analysis, we obtain the bifurcation of phase portraits of system (7) in Figure 1.

\section{Main Results and the Theoretic Derivations of the Main Results}

In this section, we state our results about solitary wave solutions, singular solutions, periodic singular solutions, and kink-shaped solutions for the first component of system (7). To relate conveniently, we omit $\varphi=\phi+c$ and the expression of the second component of system (7), that is, $\psi=g+c \varphi-$ $(1 / 2) \varphi^{2}$, in the following theorem.

Theorem 1. For given constants $c$ and $\alpha(1<\alpha<3)$, which will be given later, the Broer-Kaup equation (1) has the following exact explicit nonlinear wave solutions.

(1) When $G=c$, one obtains two kink-shaped solutions

$$
\phi_{1^{ \pm}}(x, t)= \pm \sqrt{c^{2}+2 g+2} \tanh \left(\frac{\sqrt{c^{2}+2 g+2}}{2}(x-c t)\right) \text {, }
$$


two singular solutions

$\phi_{2^{ \pm}}(x, t)= \pm \sqrt{c^{2}+2 g+2} \operatorname{coth}\left(\frac{\sqrt{c^{2}+2 g+2}}{2}(x-c t)\right)$,

and four periodic singular solutions

$$
\begin{aligned}
\phi_{3^{ \pm}}(x, t)= & \pm \sqrt{2\left(c^{2}+2 g+2\right)} \\
& \times \sec \left(\sqrt{\frac{c^{2}+2 g+2}{2}}(x-c t)\right), \\
\phi_{4^{ \pm}}(x, t)= & \pm \sqrt{2\left(c^{2}+2 g+2\right)} \\
& \times \csc \left(\sqrt{\frac{c^{2}+2 g+2}{2}}(x-c t)\right) .
\end{aligned}
$$

(2) When $c<G<G_{0}+c$, one gets two solitary wave solutions

$$
\begin{aligned}
& \phi_{5^{ \pm}}(x, t) \\
& = \pm \sqrt{\frac{c^{2}+2 g+2}{\alpha}} \\
& \times\left(\sqrt{2 \alpha-2} \cosh \left(\sqrt{\frac{c^{2}+2 g+2}{\alpha} \frac{(3-\alpha)}{2}}(x-c t)\right)\right. \\
& +2 \alpha-4) \\
& \times\left(\sqrt { 2 \alpha - 2 } \operatorname { c o s h } \left(\sqrt{\frac{c^{2}+2 g+2}{\alpha} \frac{(3-\alpha)}{2}}\right.\right.
\end{aligned}
$$

two singular solutions

$$
\begin{aligned}
& \phi_{6^{ \pm}}(x, t) \\
& = \pm \sqrt{\frac{c^{2}+2 g+2}{\alpha}} \\
& \times\left(2 \cosh \left(\sqrt{\frac{c^{2}+2 g+2}{\alpha} \frac{(3-\alpha)}{2}}(x-c t)\right)\right.
\end{aligned}
$$

$$
\begin{aligned}
+\sqrt{6-2 \alpha} \sinh \left(\sqrt{\frac{c^{2}+2 g+2}{\alpha} \frac{(3-\alpha)}{2}}\right. \\
\\
\times|x-c t|)+4-2 \alpha)
\end{aligned}
$$

$$
\begin{array}{r}
\times\left(2 \cosh \left(\sqrt{\frac{c^{2}+2 g+2}{\alpha} \frac{(3-\alpha)}{2}}(x-c t)\right)\right. \\
+\sqrt{6-2 \alpha} \sinh \left(\sqrt{\frac{c^{2}+2 g+2}{\alpha} \frac{(3-\alpha)}{2}}\right.
\end{array}
$$

$$
\times|x-c t|)-2)^{-1}
$$

and two periodic singular solutions

$\phi_{7^{ \pm}}(x, t)$

$$
\begin{aligned}
&= \pm\left(\phi _ { 2 } ( \phi _ { 6 } - \phi _ { 7 } ) \operatorname { s i n } \left(\arcsin \left(\frac{2 \phi_{2}-\phi_{6}-\phi_{7}}{\phi_{6}-\phi_{7}}\right)\right.\right. \\
&-\frac{\sqrt{-\left(\phi_{2}-\phi_{6}\right)\left(\phi_{2}-\phi_{7}\right)}}{2} \\
&\times|x-c t|) \\
&\left.+2 \phi_{6} \phi_{7}-\phi_{2} \phi_{6}-\phi_{2} \phi_{7}\right) \\
& \times\left(( \phi _ { 6 } - \phi _ { 7 } ) \operatorname { s i n } \left(\arcsin \left(\frac{2 \phi_{2}-\phi_{6}-\phi_{7}}{\phi_{6}-\phi_{7}}\right)\right.\right. \\
& \quad-\frac{\sqrt{-\left(\phi_{2}-\phi_{6}\right)\left(\phi_{2}-\phi_{7}\right)}}{2} \\
&\left.\times|x-c t|)^{-1}\right)
\end{aligned}
$$

where $\phi_{2}, \phi_{6}$, and $\phi_{7}$ will be given in the proof of the theorem.

(3) When $G=G_{0}+c$, one obtains four singular solutions as follows:

$$
\begin{aligned}
\phi_{8^{ \pm}}(x, t)= & \pm \sqrt{\frac{c^{2}+2 g+2}{3}} \\
& \times \frac{\left(1+\sqrt{\left(c^{2}+2 g+2\right) / 3}|x-c t|\right)^{2}+3}{\left(1+\sqrt{\left(c^{2}+2 g+2\right) / 3}|x-c t|\right)^{2}-1}
\end{aligned}
$$




$$
\begin{aligned}
\phi_{9^{ \pm}}(x, t)= & \pm \sqrt{\frac{c^{2}+2 g+2}{3}} \\
& \times \frac{\left(c^{2}+2 g+2\right)(x-c t)^{2}+9}{\left(c^{2}+2 g+2\right)(x-c t)^{2}-3} .
\end{aligned}
$$

Proof. (1) When $G=c$, we consider the following two kinds of orbits.

(i) First, we see that there are two heteroclinic orbits $\Gamma_{1}^{ \pm}$connected as two saddle points $\left(\phi_{0}, 0\right)$ and $\left(-\phi_{0}, 0\right)$ from Figure 1. In $(\phi, y)$-plane, from (8), the expressions of the heteroclinic orbits are given as

$$
y= \pm \frac{1}{2}\left(\phi_{0}^{2}-\phi^{2}\right) \text {. }
$$

Substituting (21) into the first equation of system (7) and integrating along the heterclinic orbits, it follows that

$$
\begin{gathered}
\int_{0}^{\phi} \frac{\mathrm{d} s}{\left(\phi_{0}^{2}-s^{2}\right)}=\frac{1}{2}|\xi|, \\
\int_{\phi}^{+\infty} \frac{\mathrm{d} s}{\left(s^{2}-\phi_{0}^{2}\right)}=\frac{1}{2}|\xi| .
\end{gathered}
$$

From (22), we have

$$
\begin{aligned}
& \phi= \pm \sqrt{c^{2}+2 g+2} \tanh \left(\frac{\sqrt{c^{2}+2 g+2}}{2} \xi\right), \\
& \phi= \pm \sqrt{c^{2}+2 g+2} \operatorname{coth}\left(\frac{\sqrt{c^{2}+2 g+2}}{2} \xi\right) .
\end{aligned}
$$

Noting that $\phi=\phi(\xi)$ and $\xi=x-c t$, we get two kinkshaped solutions $\phi_{1^{ \pm}}(x, t)$ and two singular solutions $\phi_{2^{ \pm}}(x, t)$ as (14) and (15).

(ii) Second, from the phase portrait in Figure 1, we note that there are two special orbits $\Gamma_{2}^{ \pm}$, which have the same Hamiltonian as that of the center point $(0,0)$. In $(\phi, y)$-plane, from (8), the expressions of these two orbits are given as

$$
y= \pm \frac{1}{2} \phi \sqrt{\phi^{2}-2 \phi_{0}^{2}} .
$$

Substituting (24) into the first equation of system (7) and integrating along the two orbits $\Gamma_{2}^{ \pm}$, it follows that

$$
\int_{\phi}^{+\infty} \frac{\mathrm{d} s}{s \sqrt{s^{2}-2 \phi_{0}^{2}}}=\frac{1}{2}|\xi|
$$

From (25), we have

$$
\phi= \pm \sqrt{2\left(c^{2}+2 g+2\right)} \sec \left(\sqrt{\frac{c^{2}+2 g+2}{2} \xi}\right) .
$$

At the same time, we note that if $\phi=\phi(\xi)$ is a solution of system, then $\phi=\phi(\xi+\gamma)$ is also a solution of system. Specially, we take $\gamma=\pi / 2$; we obtain another two solutions

$$
\phi= \pm \sqrt{2\left(c^{2}+2 g+2\right)} \csc \left(\sqrt{\frac{c^{2}+2 g+2}{2} \xi}\right) .
$$

Noting that $\phi=\phi(\xi)$ and $\xi=x-c t$, we get four periodic singular solutions $\phi_{3^{ \pm}}(x, t)$ and $\phi_{4^{ \pm}}(x, t)$ as (16).

(2) When $c<G<G_{0}+c$, we set the largest solution of $f(\phi)=0$ to be $\phi_{1}=\sqrt{\left(c^{2}+2 g+2\right) / \alpha}(1<\alpha<3)$, and then we can obtain another two solutions of $f(\phi)=0$ as follows:

$$
\begin{aligned}
& \phi_{2}=\frac{1}{2} \sqrt{\frac{c^{2}+2 g+2}{\alpha}}(\sqrt{4 \alpha-3}-1), \\
& \phi_{3}=-\frac{1}{2} \sqrt{\frac{c^{2}+2 g+2}{\alpha}}(\sqrt{4 \alpha-3}+1) .
\end{aligned}
$$

(i) First, we see that there is a homoclinic orbit $\Gamma_{3}$, which passes the saddle point $\left(\phi_{1}, 0\right)$. In $(\phi, y)$-plane, from $(8)$, the expressions of the homoclinic orbit are given as

$$
y= \pm \frac{1}{2} \sqrt{\left(\phi_{1}-\phi\right)^{2}\left(\phi-\phi_{4}\right)\left(\phi-\phi_{5}\right)}
$$

where

$$
\begin{aligned}
& \phi_{4}=\sqrt{\frac{c^{2}+2 g+2}{\alpha}}(\sqrt{2 \alpha-2}-1), \\
& \phi_{5}=-\sqrt{\frac{c^{2}+2 g+2}{\alpha}}(\sqrt{2 \alpha-2}+1) .
\end{aligned}
$$

Substituting (29) into the first equation of system (7) and integrating along the homoclinic orbit, it follows that

$$
\begin{gathered}
\int_{\phi_{4}}^{\phi} \frac{\mathrm{d} s}{\left(\phi_{1}-s\right) \sqrt{\left(s-\phi_{4}\right)\left(s-\phi_{5}\right)}}=\frac{1}{2}|\xi|, \\
\int_{\phi}^{+\infty} \frac{\mathrm{d} s}{\left(s-\phi_{1}\right) \sqrt{\left(s-\phi_{4}\right)\left(s-\phi_{5}\right)}}=\frac{1}{2}|\xi| .
\end{gathered}
$$

From (31), we have

$$
\begin{aligned}
\phi= & \pm \sqrt{\frac{c^{2}+2 g+2}{\alpha}} \\
& \times\left(\sqrt{2 \alpha-2} \cosh \left(\sqrt{\frac{c^{2}+2 g+2}{\alpha} \frac{(3-\alpha)}{2} \xi}\right)\right. \\
& +2 \alpha-4)
\end{aligned}
$$




$$
\begin{aligned}
\times & \left(\sqrt{2 \alpha-2} \cosh \left(\sqrt{\frac{c^{2}+2 g+2}{\alpha} \frac{(3-\alpha)}{2} \xi}\right)+2\right)^{-1}, \\
\phi= \pm & \sqrt{\frac{c^{2}+2 g+2}{\alpha}} \\
\times & \left(2 \cosh \left(\sqrt{\frac{c^{2}+2 g+2}{\alpha} \frac{(3-\alpha)}{2} \xi}\right)\right. \\
& +\sqrt{6-2 \alpha} \sinh \left(\sqrt{\frac{c^{2}+2 g+2}{\alpha}} \frac{(3-\alpha)}{2}|\xi|\right) \\
& +4-2 \alpha) \\
\times & \left(2 \cosh \left(\sqrt{\frac{c^{2}+2 g+2}{\alpha} \frac{(3-\alpha)}{2} \xi}\right)\right. \\
& +\sqrt{6-2 \alpha} \sinh \left(\sqrt{\frac{c^{2}+2 g+2}{\alpha} \frac{(3-\alpha)}{2}}|\xi|\right) \\
& -2 \\
& \cdot
\end{aligned}
$$

Noting that $\phi=\phi(\xi)$ and $\xi=x-c t$, we get two solitary solutions $\phi_{5^{ \pm}}(x, t)$ and two singular solutions $\phi_{6^{ \pm}}(x, t)$ as $(17)$ and (18).

(ii) Second, from the phase portrait in Figure 1, we note that there are another two special orbits $\Gamma_{4}^{ \pm}$, which have the same Hamiltonian as that of the center point $\left(\phi_{2}, 0\right)$. In $(\phi, y)$ plane, from (8), the expressions of these two orbits are given as

$$
y= \pm \frac{1}{2} \sqrt{\left(\phi-\phi_{2}\right)^{2}\left(\phi-\phi_{6}\right)\left(\phi-\phi_{7}\right)}
$$

where

$$
\begin{aligned}
& \phi_{6}=-\phi_{2}+\sqrt{2\left(c^{2}+2 g+2\right)-2 \phi_{2}^{2}}, \\
& \phi_{7}=-\phi_{2}-\sqrt{2\left(c^{2}+2 g+2\right)-2 \phi_{2}^{2}} .
\end{aligned}
$$

Substituting (33) into the first equation of system (7) and integrating along these two special orbits $\Gamma_{4}^{ \pm}$, it follows that

$$
\int_{\phi}^{+\infty} \frac{\mathrm{d} s}{\left(s-\phi_{2}\right) \sqrt{\left(s-\phi_{6}\right)\left(s-\phi_{7}\right)}}=\frac{1}{2}|\xi| .
$$

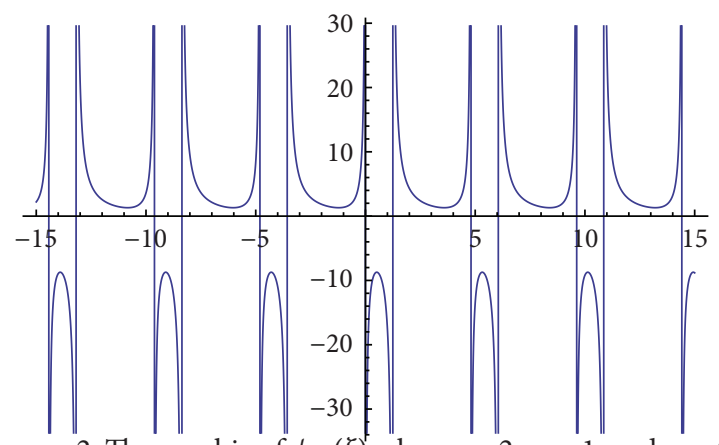

FIGURE 2: The graphic of $\phi_{8^{+}}(\xi)$ when $c=2, g=1$, and $\alpha=2$.

From (35), we have

$$
\begin{array}{r}
\phi= \pm\left(\phi _ { 2 } ( \phi _ { 6 } - \phi _ { 7 } ) \operatorname { s i n } \left(\arcsin \left(\frac{2 \phi_{2}-\phi_{6}-\phi_{7}}{\phi_{6}-\phi_{7}}\right)\right.\right. \\
\left.-\frac{\sqrt{-\left(\phi_{2}-\phi_{6}\right)\left(\phi_{2}-\phi_{7}\right)}}{2}|\xi|\right) \\
\left.+2 \phi_{6} \phi_{7}-\phi_{2} \phi_{6}-\phi_{2} \phi_{7}\right) \\
\times\left(( \phi _ { 6 } - \phi _ { 7 } ) \operatorname { s i n } \left(\arcsin \left(\frac{2 \phi_{2}-\phi_{6}-\phi_{7}}{\phi_{6}-\phi_{7}}\right)\right.\right. \\
\left.\quad-\frac{\sqrt{-\left(\phi_{2}-\phi_{6}\right)\left(\phi_{2}-\phi_{7}\right)}}{2}|\xi|\right) \\
\left.-4 \phi_{2}\right)^{-1} \cdot
\end{array}
$$

Noting that $\phi=\phi(\xi)$ and $\xi=x-c t$, we get two periodic singular solutions $\phi_{7^{ \pm}}(x, t)$ as (19).

To illustrate, we give the graphic of $\phi_{8^{+}}(\xi)$ in Figure 2 by taking $c=2, g=1$, and $\alpha=2$.

(3) When $G=G_{0}+c$, from the phase portrait in Figure 1, we note that there are two orbits $\Gamma_{5}^{ \pm}$, which have the same Hamiltonian as the degenerate saddle point $\left(\phi_{8}, 0\right)$. In $(\phi, y)$ plane, from (8), the expressions of these two orbits are given as

$$
y= \pm \frac{1}{2} \sqrt{\left(\phi-\phi_{8}\right)^{3}\left(\phi+3 \phi_{8}\right)},
$$

where

$$
\phi_{8}=\sqrt{\frac{1}{3}\left(c^{2}+2 g+2\right)} .
$$

Substituting (37) into the first equation of system (7) and integrating along these two orbits $\Gamma_{5}^{ \pm}$, it follows that

$$
\int_{\phi}^{+\infty} \frac{\mathrm{d} s}{\left(s-\phi_{8}\right) \sqrt{\left(s-\phi_{8}\right)\left(s+3 \phi_{8}\right)}}=\frac{1}{2}|\xi|,
$$




$$
\int_{\phi}^{-3 \phi_{8}} \frac{\mathrm{d} s}{\left(\phi_{8}-s\right) \sqrt{\left(\phi_{8}-s\right)\left(-3 \phi_{8}-s\right)}}=\frac{1}{2}|\xi| .
$$

From (3), we have

$$
\begin{gathered}
\phi= \pm \sqrt{\frac{c^{2}+2 g+2}{3} \frac{\left(1+\sqrt{\left(c^{2}+2 g+2\right) / 3}|\xi|\right)^{2}+3}{\left(1+\sqrt{\left(c^{2}+2 g+2\right) / 3}|\xi|\right)^{2}-1}} \\
\phi= \pm \sqrt{\frac{c^{2}+2 g+2}{3}} \frac{\left(c^{2}+2 g+2\right)(x-c t)^{2}+9}{\left(c^{2}+2 g+2\right)(x-c t)^{2}-3}
\end{gathered}
$$

Noting that $\phi=\phi(\xi)$ and $\xi=x-c t$, we get four singular solutions $\phi_{8^{ \pm}}(x, t)$ and $\phi_{9^{ \pm}}(x, t)$ as $(20)$.

Remark 2. One may find that we only consider some special orbits in Figure 1 when $G \geq c$. In fact, we may obtain exactly the same results when $G<c$.

Remark 3. We employ the software Mathematica to check the correctness of the above nonlinear wave solutions. To illustrate, we show the commands of verifying $\phi_{8^{+}}(x, t)$,

$$
\begin{aligned}
& \phi_{2}=\frac{1}{2} \sqrt{\frac{c^{2}+2 g+2}{\alpha}}(\sqrt{4 \alpha-3}-1) \text {, } \\
& \phi_{6}=-\phi_{2}+\sqrt{2\left(c^{2}+2 g+2\right)-2\left(\phi_{2}\right)^{2}} \text {, } \\
& \phi_{7}=-\phi_{2}-\sqrt{2\left(c^{2}+2 g+2\right)-2\left(\phi_{2}\right)^{2}} \text {, } \\
& \phi=\left(\phi _ { 2 } ( \phi _ { 6 } - \phi _ { 7 } ) \operatorname { S i n } \left[\operatorname{ArcSin}\left[\frac{2 \phi_{2}-\phi_{6}-\phi_{7}}{\phi_{6}-\phi_{7}}\right]\right.\right. \\
& \left.-\frac{\sqrt{-\left(\phi_{2}-\phi_{6}\right)\left(\phi_{2}-\phi_{7}\right)}}{2}(x-c t)\right] \\
& \left.+2 \phi_{6} \phi_{7}-\phi_{2} \phi_{6}-\phi_{2} \phi_{7}\right) \\
& \times\left(\phi_{6}-\phi_{7}\right) \operatorname{Sin}\left[\operatorname{ArcSin}\left[\frac{2 \phi_{2}-\phi_{6}-\phi_{7}}{\phi_{6}-\phi_{7}}\right]\right. \\
& \left.-\frac{\sqrt{-\left(\phi_{2}-\phi_{6}\right)\left(\phi_{2}-\phi_{7}\right)}}{2}(x-c t)\right] \\
& \left.-4 \phi_{2}\right)^{-1}
\end{aligned}
$$

$$
\begin{gathered}
\varphi=\phi+c \\
\psi=g+c \varphi-\frac{1}{2} \varphi^{2}, \\
\text { Simplify }\left[\partial_{t} \varphi+\partial_{x} \psi+\frac{1}{2} \partial_{x}\left(\varphi^{2}\right)\right], \\
\text { Simplify }\left[\partial_{t} \psi+\partial_{x}\left(\varphi \psi+\varphi+\partial_{x, x} \varphi\right)\right] .
\end{gathered}
$$

\section{Conclusions}

In this paper, by employing the bifurcation method and qualitative theory of dynamical systems, we study the nonlinear wave solutions for the Broer-Kaup equation (1) and obtain exact explicit expressions of the various kinds of nonlinear wave solutions, which include solitary wave solutions, singular solutions, periodic singular solutions, and kink-shaped solutions. To the best of our knowledge, most of the nonlinear wave solutions are newly obtained.

\section{Conflict of Interests}

The author declares that there is no conflict of interests regarding the publication of this paper.

\section{Acknowledgments}

This research was supported by Promotion Program for Young and Middle-Aged Teacher in Science and Technology Research of Huaqiao University (no. ZQN-PY119) and the Foundation of Huaqiao University (no. 12BS223).

\section{References}

[1] L. J. F. Broer, "Approximate equations for long water waves," Applied Scientific Research, vol. 31, no. 5, pp. 377-395, 1975.

[2] D. J. Kaup, "A higher-order water-wave equation and the method for solving it," Progress of Theoretical Physics, vol. 54, no. 2, pp. 396-408, 1975.

[3] B. A. Kupershmidt, "Mathematics of dispersive water waves," Communications in Mathematical Physics, vol. 99, no. 1, pp. 5173, 1985.

[4] M. Ablowitz and H. Segur, "Solitons, nonlinear evolution equations and inverse scattering," Journal of Fluid Mechanics, vol. 244, pp. 721-725, 1992.

[5] M. Wang, J. Zhang, and X. Li, "Application of the $G^{\prime} / G$ expansion to travelling wave solutions of the Broer-Kaup and the approximate long water wave equations," Applied Mathematics and Computation, vol. 206, no. 1, pp. 321-326, 2008.

[6] D. Ganji, H. B. Rokni, M. Sfahani, and S. Ganji, "Approximate traveling wave solutions for coupled whitham-broer-kaup shallow water," Advances in Engineering Software, vol. 41, no. 7, pp. 956-961, 2010.

[7] S. Guo, Y. Zhou, and C. Zhao, "The improved $G^{\prime} / G$-expansion method and its applications to the Broer-Kaup equations and approximate long water wave equations," Applied Mathematics and Computation, vol. 216, no. 7, pp. 1965-1971, 2010. 
[8] M. Alquran, K. Al-Khaled, and H. Ananbeh, "New soliton solutions for systems of nonlinear evolution equations by the rational sine-cosine method," Studies in Mathematical Sciences, vol. 3, no. 1, pp. 1-9, 2011.

[9] M. F. El-Sabbagh and S. I. El-Ganaini, "The He's variational principle to the Broer-Kaup (BK) and Whitham Broer-Kaup (WBK) systems," International Mathematical Forum, vol. 7, no. 41-44, pp. 2131-2141, 2012.

[10] M. F. El-Sabbagh and S. I. El-Ganaini, "New exact solutions of Broer-Kaup (BK) and Whitham Broer-Kaup (WBK)systems via the first integral method," International Journal of Mathematical Analysis, vol. 6, no. 45-48, pp. 2287-2298, 2012.

[11] M. Song, S. Li, and J. Cao, "New exact solutions for the $(2+$ 1)-dimensional Broer-Kaup-Kupershmidt equations," Abstract and Applied Analysis, vol. 2010, Article ID 652649, 9 pages, 2010.

[12] Z. Wen, Z. Liu, and M. Song, "New exact solutions for the classical drinfel'd-sokolov-Wilson equation," Applied Mathematics and Computation, vol. 215, no. 6, pp. 2349-2358, 2009.

[13] Z. Wen and Z. Liu, "Bifurcation of peakons and periodic cusp waves for the generalization of the Camassa-Holm equation," Nonlinear Analysis. Real World Applications, vol. 12, no. 3, pp. 1698-1707, 2011.

[14] B. He, Q. Meng, J. Zhang, and Y. Long, "Periodic loop solutions and their limit forms for the Kudryashov-Sinelshchikov equation," Mathematical Problems in Engineering, vol. 2012, Article ID 320163, 10 pages, 2012.

[15] Z. Wen, "Extension on bifurcations of traveling wave solutions for a two-component Fornberg-Whitham equation," Abstract and Applied Analysis, vol. 2012, Article ID 704931, 15 pages, 2012.

[16] Z. Wen, "Bifurcation of traveling wave solutions for a twocomponent generalized $\theta$-equation," Mathematical Problems in Engineering, vol. 2012, Article ID 597431, 17 pages, 2012.

[17] S. Li and Z. Liu, "The traveling wave solutions and their bifurcations for the bbm-like $B(m, n)$ equations," Journal of Applied Mathematics, vol. 2013, Article ID 490341, 17 pages, 2013.

[18] Y. Long and C. Chen, "Existence analysis of traveling wave solutions for a generalization of $\mathrm{KdV}$ equation," Mathematical Problems in Engineering, vol. 2013, Article ID 462957, 7 pages, 2013.

[19] M. Song, B. S. Ahmed, and A. Biswas, "Topological soliton solution and bifurcation analysis of the Klein-Gordon-Zakharov equation in $(1+1)$-dimensions with power law nonlinearity," Journal of Applied Mathematics, vol. 2013, Article ID 972416, 7 pages, 2013.

[20] Y. Wu and Z. Liu, "New types of nonlinear waves and bifurcation phenomena in Schamel-Korteweg-de Vries equation," Abstract and Applied Analysis, vol. 2013, Article ID 483492, 18 pages, 2013.

[21] Z. Wen, "Bifurcation of solitons, peakons and periodic cusp waves for $\theta$-equation," Nonlinear Dynamics, 2014. 


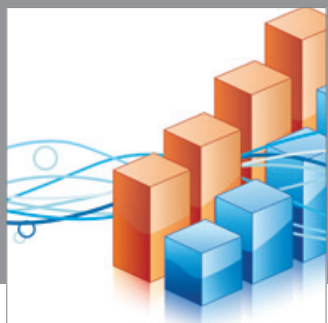

Advances in

Operations Research

mansans

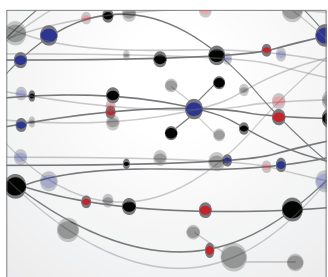

The Scientific World Journal
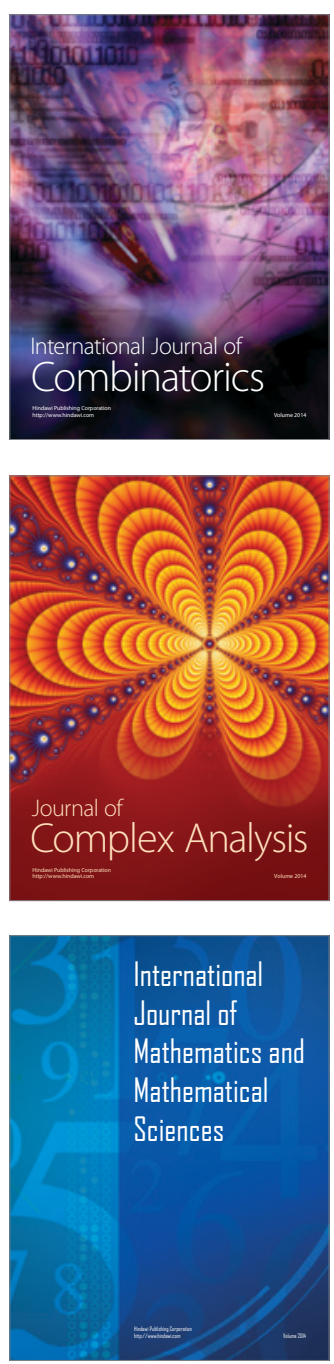
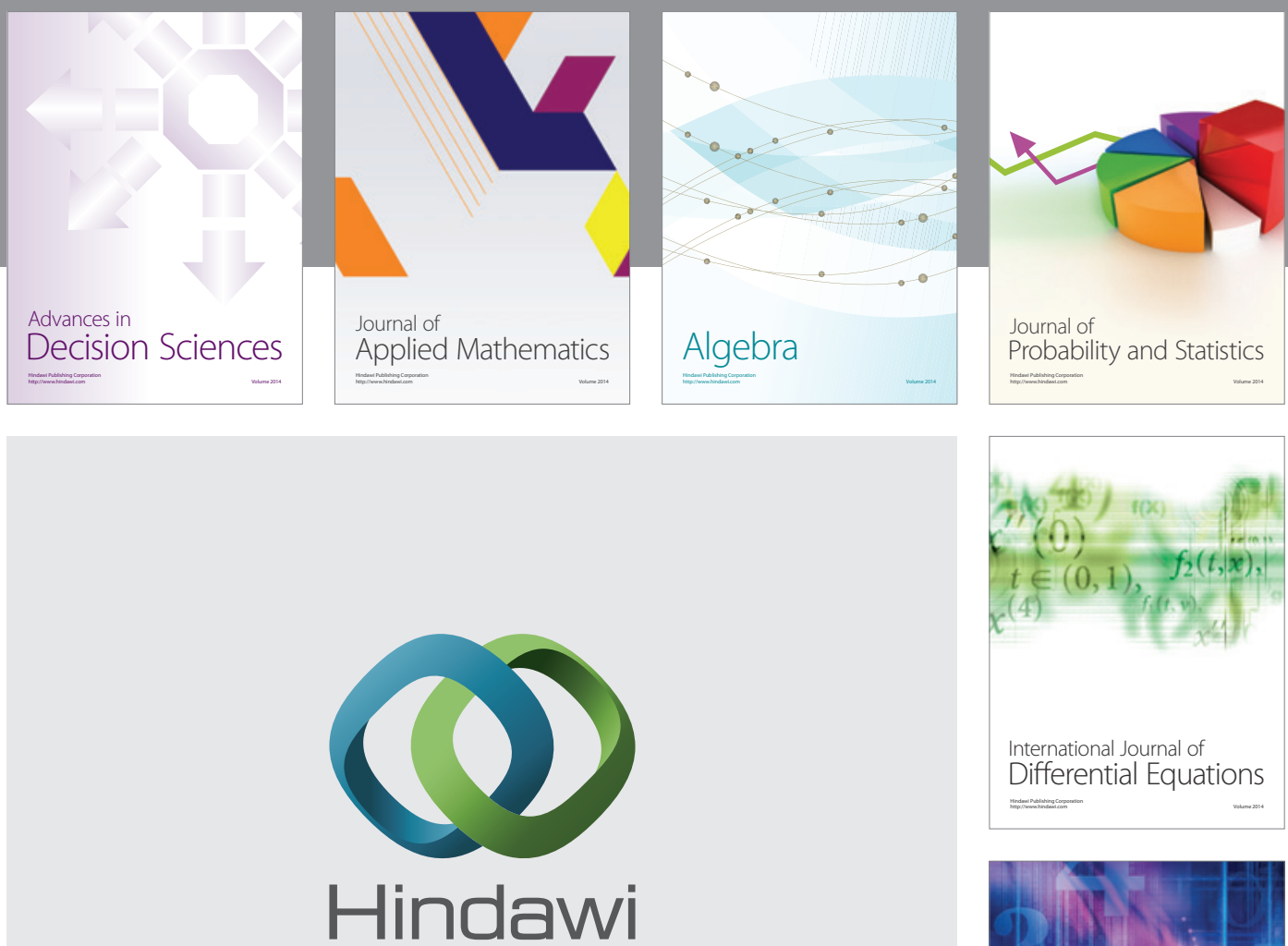

Submit your manuscripts at http://www.hindawi.com
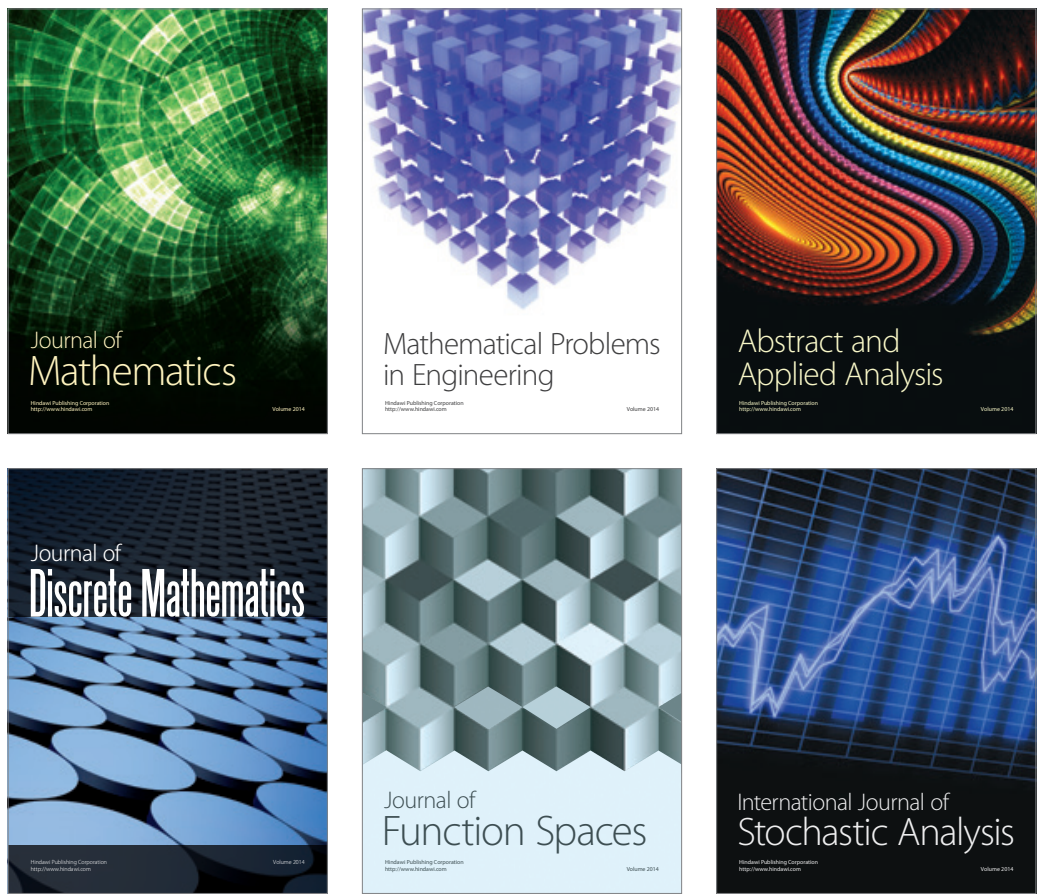

Journal of

Function Spaces

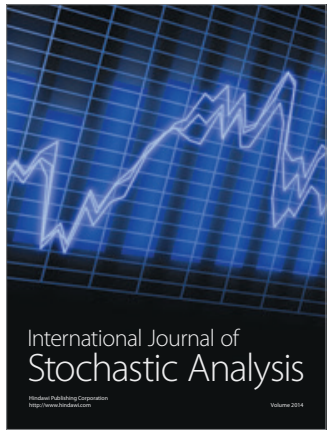

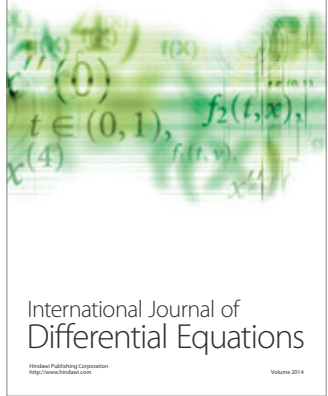
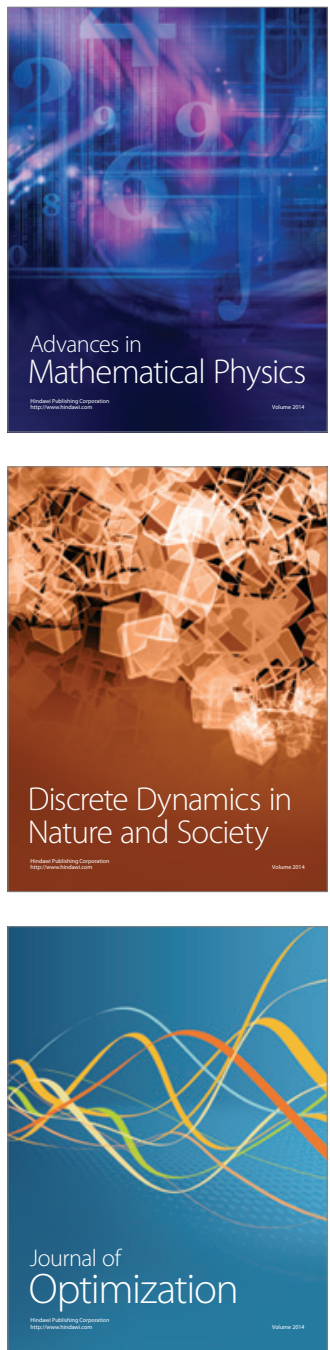